Goemans, A. F., Spence, S. J. and Ramsey, I. K. (2017) Validation and determination of a reference interval for Canine HbA1c using an immunoturbidimetric assay. Veterinary Clinical Pathology, 46(2), pp. 227-237. (doi: $10.1111 / \mathrm{vcp} .12477$ )

There may be differences between this version and the published version. You are advised to consult the publisher's version if you wish to cite from it.

This is the peer-reviewed version of the following article: Goemans, A. F., Spence, S. J. and Ramsey, I. K. (2017) Validation and determination of a reference interval for Canine HbA1c using an immunoturbidimetric assay. Veterinary Clinical Pathology, 46(2), pp. 227-237, which has been published in final form at 10.1111/vcp.12477. This article may be used for non-commercial purposes in accordance with Wiley Terms and Conditions for Self-Archiving.

http://eprints.gla.ac.uk/128386/

Deposited on: 16 September 2016

Enlighten - Research publications by members of the University of Glasgow http://eprints.gla.ac.uk 
1 Title :Validation and Determination of a Reference Interval

2 for Canine HbA1c Using an Immunoturbidimetric Assay

4 Short Title: Validation of a method for measuring canine HbA1c

5

6 Authors:

$7 \quad$ Anne F Goemans ${ }^{1}$

8 Susanna J Spence ${ }^{1}$

$9 \quad$ lan K Ramsey ${ }^{1}$

10

11 Affilitiations: ${ }^{1}$ University of Glasgow

12

13 Corresponding author:

14 Professor lan K Ramsey,

15 Small Animal Hospital

16 University of Glasgow

17 Bearsden Road, Bearsden

18 Glasgow G61 1QH UK

19 email: Ian.Ramsey@glasgow.ac.uk 
21 Abstract

22 Background: Hemoglobin A1c $(\mathrm{HbA1c})$, provides a reliable measure of glycemic control over 2 to 3 months in human diabetes mellitus (DM). In dogs, formation of $\mathrm{HbA1c}$ has been demonstrated, but there are no validated commercial assays.

Objective: To validate a commercially available automated immunoturbidimetric assay for canine $\mathrm{HbA} 1 \mathrm{c}$ and determine a reference interval in a hospital population of various ages and breeds.

Methods: The specificity of the assay was assessed by inducing glycosylation in vitro using isolated canine hemoglobin. Repeatability was assessed by measuring canine samples 5 times in succession, long term inter-assay imprecision by measuring supplied control materials, stability using samples stored at $4^{\circ} \mathrm{C}$ over 5 days and $-20^{\circ} \mathrm{C}$ over 8 weeks, linearity by mixing samples of known $\mathrm{HbA} 1 \mathrm{C}$ in differing proportions, and the effect of anticoagulants by taking paired samples. A reference interval was determined using EDTA-anticoagulated blood samples from 60 non-diabetic hospitalised animals of various ages and breeds. HbA1c was also measured in dogs with DM $(n=10)$.

Results: HbA1c increased proportionally with glucose concentration in vitro. The mean repeatability was $4.1 \%$ (range $1.2 \%-6.1 \%$ ). Samples were stable for 5 days at $4^{\circ} \mathrm{C}$. The assay was linear within the assessed range. EDTA- and heparinanticoagulated blood can be used interchangeably for $\mathrm{HbA} 1 \mathrm{c}$ measurement. The reference interval for $\mathrm{HbA} 1 \mathrm{c}$ was $9-18.5 \mathrm{mmol} / \mathrm{mol}$. There was no apparent effect of age or breed on HbA1c. HbA1c ranged from $14-48 \mathrm{mmol} / \mathrm{mol}$ in dogs with DM.

Conclusions: The assay provides a reliable method of canine $\mathrm{HbA} 1 \mathrm{c}$ measurement with good analytical performance. 
46 Key words: analytical performance, diabetes mellitus, dogs, glycosylated

47 hemoglobin, method validation

\section{Abbreviations used}

50 ANOVA - analysis of variance

$51 \quad$ CBC - complete blood count

$52 \mathrm{Cl}$ - confidence interval

53 CV - coefficient of variance

54 DM - diabetes mellitus

55 EDTA - ethylenediaminetetraacetic acid

$56 \mathrm{HbA1c}-$ hemoglobin A1c

57 RCF - relative centrifugal force

58 SD - standard deviation

\section{Introduction}

62 Glycosylated hemoglobin A1c (HbA1c) is formed when glucose binds to the $n$ terminal valine of the $\beta$-subunit of hemoglobin $A$ and makes up the largest fraction of the total glycosylated hemoglobin $(\mathrm{HbA} 1)^{1}$. The total amount of $\mathrm{HbA} 1 \mathrm{c}$ formed is dependent on erythrocyte lifespan, erythrocyte permeability to glucose and the

66 average blood glucose concentration throughout that erythrocyte lifespan ${ }^{1}$. In people

67 it is related to the average blood glucose concentration over the preceding 2-3 
months, weighted towards the most recent 2-4 weeks ${ }^{2}$. Fructosamine - glycosylated plasma proteins - are currently a commonly used measure of longer term diabetic stability in the dog. Fructosamine are related to the average blood glucose concentration over the previous 1-2 weeks ${ }^{3,4}$. However a number of factors other than plasma glucose concentration affect fructosamine concentration including hypoproteinemia, hyperlipidemia and azotemia $^{4}$ Compared to fructosamine measurements, $\mathrm{HbA1c}$ is less affected by pathological conditions other than $\mathrm{DM}^{5}$.

In people $\mathrm{HbA1c}$ has shown itself to be a highly specific and reliable biomarker for the long term control of both type $1^{6}$ and type $2 \mathrm{DM}^{7}$. HbA1c is also used prognostically with increasing values corresponding to increased risk of diabetic complications $^{6}$. In dogs, there have been no studies looking at the relationship between fructosamine or $\mathrm{HbA} 1 \mathrm{c}$ and outcome in $\mathrm{DM}$.

The measurement of $\mathrm{HbA} 1 \mathrm{c}$ in dogs has been previously described. Several methods developed for human use have been evaluated for use in canine DM but none have been adopted into standard clinical practice. All published studies have however shown that canine diabetics have higher average ratios of glycosylated hemoglobin: total hemoglobin compared to non-diabetic controls ${ }^{3,8-18}$.

The first aim of this study was to determine the effect of increasing glucose concentrations on the production of canine $\mathrm{HbA} 1 \mathrm{c}$ as measured by an immunoturbidimetric assay, the Siemens DCA ${ }^{\mathrm{TM}}$ Vantage (Siemens Healthcare plc. Surrey, UK), in canine erythrocyte preparations. The second aim was to validate this method and to establish a reference interval from a hospital population. The final aim was to assess the effect of breed pre-disposition and age on canine HbA1c within the reference population. 
Materials and Methods

94

\section{Animals and Samples}

Specimens obtained were all taken from dogs referred to the Small Animal Hospital, University of Glasgow. Ethical approval for this study was obtained from the School of Veterinary Medicine Ethics and Welfare committee, University of Glasgow.

Hospital Population

100 Surplus EDTA-anticoagulated and heparin-anticoagulated blood from samples that had been taken for diagnostic purposes by jugular venipuncture were used for the method validation aspect of the study. Screening for eligibility into the reference interval occurred between June and August 2015 with 60 samples meeting all criteria selected. The inclusion criteria were a record of a CBC measured using a Cell-Dyn hematology analyzer (Abbott Laboratories, North Chicago, IL, USA) with WBC differential counts performed manually, and biochemistry analyses including a near normal plasma glucose concentration of $3-7 \mathrm{mmol} / \mathrm{L}(54-126 \mathrm{mg} / \mathrm{dL})$ measured by the hexokinase G-6-PDH method in a fluoride oxalate-anticoagulated sample within 8 hours using an Olympus AU640 biochemistry analyzer (Olympus

110 Corporation, Tokyo, Japan). As part of the biochemistry analyses cholesterol (by combined cholesterol esterase/oxidase method) and triglycerides (by combined lipolysis/ glycerol kinase/ glycerol phosphate oxidase method) were also measured on the Olympus AU640. Exclusion criteria were a diagnosis of DM or other endocrine disorders. Grossly hemolyzed and lipemic samples were excluded. Animals with a hemoglobin concentration $<12 \mathrm{~g} / \mathrm{dL}$ were excluded from the reference interval 
population as anemia has been shown to have an effect on HbA1c in previous

117 canine studies ${ }^{13} 16$. Animals with diseases unrelated to carbohydrate metabolism were not excluded. Information was collected on the animal's age; breed, sex, comorbidities, current medication, blood glucose, and hemoglobin as well as any abnormalities found on hematology and biochemistry. To ensure a wide range of dogs were included in the reference interval population, dogs were prospectively recruited into 4 similarly sized sub-groups based on age and diabetic predisposition; young ( $=<5$ years old) non-predisposed, older (>5 years old) non-predisposed, young predisposed, and older predisposed. The diabetic predisposition was based on the list as shown in the supplementary information.

\section{Diabetic population}

127 Surplus heparin-anticoagulated and EDTA-anticoagulated blood samples, taken for 128 monitoring purposes were also available for 10 diabetic dogs receiving insulin therapy. Diagnosis had been confirmed before sample collection on the basis of consistent clinical signs of polyuria, polydipsia and weight loss, concurrent hyperglycemia and glucosuria. Information was collected on age, breed, sex, comorbidities, medications, blood glucose, hemoglobin and any hematological and biochemical abnormalities as well as any serum fructosamine measurements performed using the Nitrotetrazolium blue reduction method on an Olympus AU640 biochemistry analyser with $\mathrm{ABX}$ Horiba reagent kit (Horiba UK Ltd - Medical, Northampton, UK) as part of routine monitoring.

\section{Analysis of HbA1c}

138 Samples were analysed on a $\mathrm{DCA}^{\mathrm{TM}}$ Vantage using $\mathrm{HbA} 1 \mathrm{c}$ reagent cartridges 139 (Siemens DCA ${ }^{\mathrm{TM}}$ Systems Hemoglobin A1c reagent kit) which contain all reagents in 
a single disposable cartridge that the sample is slotted into, effectively eliminating carry-over. The Siemens DCA $^{\mathrm{TM}}$ Vantage uses murine anti-human $\mathrm{HbA1c}$ monoclonal antibodies specific to the last few amino acids on the $\mathrm{N}$-terminal of the $\beta$ chain ${ }^{19}$. Canine and human hemoglobin A are only $80 \%$ homologous, but the first 5 residues of the $\mathrm{N}$-terminal of the $\beta$-chain are identical ${ }^{16}$. $\mathrm{HbA} 1 \mathrm{c}$ in the sample causes an inhibition of latex agglutination by binding competitively to the anti-HbA1c antibodies and decreasing absorbance at $531 \mathrm{~nm}$. Concurrently total hemoglobin is measured following the oxidation to methemoglobin, complexed with thiocyanate to form a colored compound which is measured spectrophotometrically at $531 \mathrm{~nm}$. $\mathrm{HbA} 1 \mathrm{c}$ is expressed as a ratio of total hemoglobin in $\mathrm{mmol} / \mathrm{mol}$ within 6 minutes. All reactions and calculations are carried out internally ${ }^{19}$.

The samples were analysed according to the manufacturers' instructions using $1 \mu \mathrm{L}$ of EDTA-anticoagulated blood. Before each batch run and when changing cartridge batch numbers a quality control run with one normal (range $24.6-48.6 \mathrm{mmol} / \mathrm{mol}$ ) and one abnormal (range $70-116.4 \mathrm{mmol} / \mathrm{mol}$ ) sample was performed using commercial control reagents (Siemens DCA ${ }^{\mathrm{TM}}$ systems).

\section{Incubation of canine hemoglobin with glucose}

EDTA-anticoagulated blood from 5 non-diabetic dogs with no hematological abnormalities was pooled. This sample was washed 3 times with phosphate buffered saline by centrifuging at a relative centrifugal force (RCF) of 1439 using a Beckman Coulter Allegra X-12R (Beckman Coulter, High Wycombe, UK) for 10 minutes. The washed erythrocytes were hemolyzed using a modified osmotic shock procedure ${ }^{20}$, then centrifuged at an RCF of 2249 for 15 minutes to sediment the cell debris. The hemolysate was divided into 5 aliquots of $600 \mu \mathrm{L}$. The hemolysates were incubated at 
glucose concentrations of $0,50,100,200$ and $400 \mathrm{mmolL}$ (equivalent to 900,1800 , 3600 and $7200 \mathrm{mg} / \mathrm{dL}$ ), stored at room temperature, and measured at days $0,7,11$, and 17 after preparation. A separate pool of intact erythrocytes was washed in the same manner and half the sample was hemolyzed. Both hemolysates and intact erythrocytes were divided into $500 \mu \mathrm{L}$ aliquots and incubated at $4^{\circ} \mathrm{C}$ in the same glucose concentrations as the room temperature hemolysates. Samples were measured at $0,4,7$ and 14 days after preparation.

\section{Repeatability}

Samples were collected as described previously from both diabetic and non-diabetic animals to represent a large range of glucose concentrations. To evaluate the repeatability, the HbA1c of each sample $(n=4)$ was measured 5 times in succession for each sample. To evaluate the inter-assay variability over the course of the study the results from the commercially available quality control materials were used.

Storage stability was evaluated using 3 EDTA-anticoagulated samples kept at $4^{\circ} \mathrm{C}$. Samples were measured at baseline and then for 4 consecutive days, with the measurements at each time point compared to baseline. Stability of canine HbA1c at $-20^{\circ} \mathrm{C}$ was assessed by freezing 5 samples, measuring these after 4 and 8 weeks and comparing to baseline.

\section{Linearity}

Samples from 2 dogs were used. To achieve a range, one set of samples was from the reference interval and the other from the diabetic population. HbA1c was measured in the EDTA-anticoagulated samples and then mixed in varying proportions $(25: 75,50: 50$ and $75: 25)$ to create 3 new intermediate expected values, 
187 which were then compared to the measured $\mathrm{HbA}_{1 \mathrm{c}^{21}}$. This was repeated with the heparin-anticoagulated samples to assess both linearity of the assay and the effect of different anti-coagulants to the measured $\mathrm{HbA1c}$.

\section{Interference}

An initial investigation into the effect of lipemia on the assay was performed. EDTAanticoagulated samples were obtained as previously described. Samples from 7 dogs with known total lipid concentrations, triglycerides from $0.68-25.58 \mathrm{mmol} / \mathrm{L}$ and cholesterol from $4.9-11.7 \mathrm{mmol} / \mathrm{L}$ were used. 4 of the samples came from diabetic dogs, 2 from non-diabetics who had been excluded from the reference interval due to gross hyperlipemia, and one control from the reference interval population. After assessing the baseline $\mathrm{HbA1c}$, the samples were centrifuged (Beckman Coulter Allegra X-12R) at $2073 \mathrm{RCF}$ at $6^{\circ} \mathrm{C}$ for 12 minutes. The plasma was completely removed and an equal volume of $0.9 \% \mathrm{NaCl}$ added. The packed red cells were re-suspended by mixing well and the samples were measured again.

To assess the effect of complete hemolysis on $\mathrm{HbA1c}$ measurements, the starting time point results from the haemolyzed preparations used in the $4^{\circ} \mathrm{C}$ glucose incubation experiments (described earlier) were compared to the results from the intact erythrocyte preparations (which were from the same pooled sample but had not undergone haemolysis).

\section{Statistical methods}

Statistical analysis was carried out in SPSS v22 (IBM) or Microsoft Excel 2010 for Windows with Reference Value Advisor Add-In ${ }^{22}$. Statistical significance was set at $p=0.05$ for all analyses. 
211 For the glucose incubation regression lines were fitted. The lines of measured

212 HbA1c over time were fitted for each glucose concentration and compared against

213 the control (0 mmolL glucose). The change in $\mathrm{HbA1c}$ from baseline was compared to

214 glucose concentration for each time point using a linear regression and Pearson

215 correlation.

216 To evaluate linearity a least squares regression analysis was performed on expected

217 against observed measurements. The difference between EDTA and heparin 218 anticoagulant was evaluated by calculating the mean difference and performing a 219 least squares regression analysis.

220 The reference interval for the hospital population was determined using the nonparametric method with bootstrapping to arrive at the $90 \%$ confidence interval for the whole population ${ }^{22}$. Both Tukey and Dixon-Reed tests were used to identify potential outliers. The reference interval comprises the central $95 \%$, with $90 \%$ confidence 224 intervals around the lower and upper limits.

225 All factors assessed - HbA1c age, breed-predisposition, glucose, hemoglobin, sex were summarised descriptively. Linear regression and correlations were performed

227 to look at the relationship between $\mathrm{HbA1c}$ and glucose concentration, age and 228 hemoglobin concentration. A regression model of $\mathrm{HbA1c}$ was fitted with age, haemoglobin concentration, sex, breed-predisposition and glucose concentration as co-factors.

231 A Kruskal-Wallis ANOVA was used to assess the overall difference in HbA1c 232 between the pre-specified reference interval groups. Subgroup analysis was performed within the reference population by comparing the median $\mathrm{HbA} 1 \mathrm{c}$ in each 
subgroup to that of the median $\mathrm{HbA1c}$ of the other subgroups combined using a

236 For the diabetic population all factors assessed were summarised descriptively and compared to the reference interval population. Normally distributed factors were compared using an independent sample t-test and non-normally distributed factors were compared using a Mann-Whitney U test.

\section{Results}

$\mathrm{HbA1c}$ synthesis in vitro at room temperature using canine hemolysates increases with time and glucose concentration (Figure 1). Using a least squares regression to fit lines for each concentration over time, the concentrations were found to be significantly different from each other $(p<0.001)$. Additionally at each time point, the change in $\mathrm{HbA1c}$ from baseline against glucose concentration gave a linear relationship; day 7 , Pearson correlation $0.968, R^{2}$ linear $0.937(p=0.007)$; day $11, R^{2}$ $0.941(p=0.006)$; day $17, R^{2} 0.941(p=0.006)$. The increase in HbA1c over time was initially linear for all glucose concentrations, but between days 11 and 17 the rate of change started to decrease. Further points were not measured. The aliquots kept at $4^{\circ} \mathrm{C}$ did not show any significant deviations from baseline after 14 days.

251 The mean co-efficient of variation using canine samples measured 5 times in succession was $4.08 \%$ (range $1.16 \%-6.10 \%$ ) (Table 1 ) with a maximum difference of $2 \mathrm{mmol} / \mathrm{mol}$ between the highest and lowest measurement within any sample.

254 One measurement of $57 \mathrm{mmol} / \mathrm{mol}$ was excluded from the analysis of the canine diabetic sample due to operator error. 
Validation of a method for measuring canine $\mathrm{HbA1c}$

256 The inter-assay imprecision over the course of the study was $2.97 \%$ with the normal

257 concentration quality control material (stabilised human hemoglobin) supplied by the manufacturer. The inter-assay imprecision with the high $\mathrm{HbA} 1 \mathrm{c}$ commercial control had a larger CV of $8.81 \%$. Bias was seen with an overestimation of the mean relative to the stated value (mean $93.5 \mathrm{mmol} / \mathrm{mol}$ ) (Table 1 ).

There was a low variation due to storage over 5 days using the canine samples with a mean $\mathrm{CV}$ of $2.12 \%$ (Table 1 ). The maximum absolute change from baseline at any time point was $1 \mathrm{mmol} / \mathrm{mol}$ with a mean change from baseline after 5 days of $0.3 \mathrm{mmol} / \mathrm{mol}$. After freezing for 4 weeks there was low variation compared to baseline with a maximum absolute change of $1 \mathrm{mmol} / \mathrm{mol}$. After 8 weeks 4 samples showed a small change from baseline with the fifth showing a decrease of 7 $\mathrm{mmol} / \mathrm{mol}(-14 \%)$ from baseline.

In the study of linearity, regression analysis of the heparin-anticoagulated and EDTAanticoagulated blood samples gave a linear relationship for both (Figure 2). $\mathrm{R}^{2}$ linear for EDTA was 0.982 and for heparin 0.957 . For EDTA samples the y intercept was $2.23 \mathrm{mmol} / \mathrm{mol}(95 \% \mathrm{Cl}-4.539$ to $0.077 \mathrm{mmol} / \mathrm{mol})$ and the slope was $1.031(95 \% \mathrm{Cl}$ 0.951 to 1.111$)$. For the heparin samples the $y$ intercept was $-1.846 \mathrm{mmol} / \mathrm{mol}(95 \%$ $\mathrm{Cl}-5.5$ to $1.8 \mathrm{mmol} / \mathrm{mol})$ and the slope $1.046(95 \% \mathrm{Cl} 0.918-1.174)$. For both lines the $y$ intercept was not significantly different from 0 , the slope was not different from 1 and the lines were not significantly different from each other $(p=0.92)$. The mean difference between the heparin and EDTA samples was $-0.8 \mathrm{mmol} / \mathrm{mol}$.

277 Regarding interference from hyperlipemia, the initial HbA1c measured ranged from 27811 to $34 \mathrm{mmol} / \mathrm{mol}$ with a trend for increasing interference from lipids as the 
triglyceride concentration increases (Table 2). The largest difference was $6 \mathrm{mmol} / \mathrm{mol}$ (60\% change from baseline for sample 5 and a $20.7 \%$ change for sample 7 ).

The HbA1c values obtained from the hemolyzed pooled sample were higher, mean $22 \mathrm{mmol} / \mathrm{mol}$, than the intact erythrocytes, mean $17.75 \mathrm{mmol} / \mathrm{mol}$, with no overlap between the two samples.

The reference population comprised of 9 predisposed breeds and 18 nonpredisposed breeds. The most common predisposed breeds were Border collies $(n=9)$, Bichon Frises $(n=3)$, Cavalier King Charles Spaniels $(n=3)$ and Yorkshire terriers $(n=2)$. The most common non-predisposed breeds were Labradors $(n=6)$, Crossbreeds ( $n=5)$, German Shepherd dogs $(n=5)$, Golden Retrievers $(n=4)$ and Cocker Spaniels $(n=4)$. The reference interval was calculated from 60 animals. No outliers were excluded. The distribution of the reference values was not normal, Shapiro-Wilk $p=0.03$, showing evidence of a binomial distribution (Figure 3). Using the non-parametric method $(n>40)$ a reference interval for HbA1c of $9-18.5$ $\mathrm{mmol} / \mathrm{mol}$ was obtained with a lower limit $90 \% \mathrm{Cl}$ of $9-10.5 \mathrm{mmol} / \mathrm{mol}$ and an upper limit $90 \% \mathrm{Cl}$ of $18-19 \mathrm{mmol} / \mathrm{mol}$. The mean value obtained was $14.3 \mathrm{mmol} / \mathrm{mol}$ $\mathrm{HbA1c}(\mathrm{SD} 2.5)$ with a total range of $9-19 \mathrm{mmol} / \mathrm{mol}$ observed for the reference population (Table 3).

There was a weak positive correlation between $\mathrm{HbA} 1 \mathrm{c}$ and plasma glucose concentration within the reference population, Spearman's $\sigma 0.332, r^{2}=0.089$. The correlations between age and $\mathrm{HbA} 1 \mathrm{c}$ and between hemoglobin and $\mathrm{HbA} 1 \mathrm{c}$ were not significant within the reference population. Within the regression model the overall adjusted $r^{2}$ was low (0.146) and the only significant factor was glucose concentration $(p=0.02)$ with an increase in $\mathrm{HbA} 1 \mathrm{c}$ of $1.259 \mathrm{mmol} / \mathrm{mol}$ for every $1 \mathrm{mmol} / \mathrm{L}$ increase 
303 in plasma glucose concentration. Both being neutered and being of a non304 predisposed breed decreased $\mathrm{HbA} 1 \mathrm{c}$ in the model (estimate $-0.8448, \mathrm{p}=0.19$ and 1.002, $p=0.12$ respectively) but neither was statistically significant. Age, hemoglobin concentration and male vs female were all non-significant factors $(p=0.09, p=0.23$, $\mathrm{p}=0.68)$.

Differences in age, sex and breed predisposition were observed between the groups

(Table 3), but only the old non-predisposed group had a higher HbA1c (mean $15.28 \mathrm{mmol} / \mathrm{mol}$ ) when compared to the rest of the reference interval (mean $13.88 \mathrm{mmol} / \mathrm{mol}) \mathrm{p}=0.045$. There were no significant differences in $\mathrm{HbA1c}$ across the sub-groups using a Kruskal-Wallis ANOVA. There were also no significant differences when partitioning by age $(p=0.442)$ or breed predisposition $(p=0.213)$ (Figure 3).

The DM group ( $n=10)$ was significantly older $(p=0.005)$ and had a higher proportion of dogs of predisposed breeds $(7 / 10)(p=0.047)$ compared to the reference interval population. As expected, the DM group had significantly higher glucose concentrations, mean $19.56 \mathrm{mmolL}$ vs. $4.9 \mathrm{mmolL}(p<0.001)$ (Table 3$)$. The HbA1c, mean $36.5 \mathrm{mmol} / \mathrm{mol}$ vs. $14.3 \mathrm{mmol} / \mathrm{mol}(\mathrm{p}<0.001)$, was also significantly higher

(Figure 4). There were no differences in hemoglobin $(p=0.454)$ and sex $(p=0.442)$ between DM group and the reference population. Within the DM group there was no significant correlation between $\mathrm{HbA} 1 \mathrm{c}$ and glucose concentration $\mathrm{p}=0.618(\mathrm{n}=9)$ or

323 fructosamine $p=0.827(n=6)$.

\section{Discussion}

Using pooled erythrocytes from non-diabetic dogs it was demonstrated that incubation with glucose increased the proportion of $\mathrm{HbA1c}$ measured in vitro with 
327 increasing glucose concentration. From the known kinetics of the reaction ${ }^{12}$ and 328 work using human erythrocyte preparations ${ }^{23}$, this increase was expected and thus suggests that the $\mathrm{DCA}^{\mathrm{TM}}$ Vantage is measuring the stable canine $\mathrm{HbA1c}$ fraction and that canine $\mathrm{HbA} 1 \mathrm{c}$ can be used to quantify the long term glycemic control of a canine patient. Using human erythrocytes it was previously shown that HbA1c increases with increasing glucose concentrations at $4^{\circ} \mathrm{C}^{23}$. However this was not replicated in our experiments, with values not deviating significantly from baseline after 14 days. The previous study used agar gel electrophoresis for detecting $\mathrm{HbA1c}$, which does not differentiate between stable and labile fractions of HbA1c. However, the reaction of hemoglobin with glucose to form labile HbA1c occurs 11.9 times faster than to stable $\mathrm{HbA}_{1 \mathrm{c}^{2}}$ and so increases were seen more quickly than in our study. The only previous study using canine erythrocyte preparations also looked at increases in labile $\mathrm{HbA} 1 \mathrm{c}$, incubating their preparations with a high glucose concentration for just 6 hours and increasing glucose concentrations were not evaluated ${ }^{18}$.

Intact erythrocytes were not used for the room temperature incubation as they are prone to extensive and variable hemolysis ${ }^{24}$. Using hemolysates controlled for this variable, but made the model less representative of in vivo conditions. We were however able to show that canine $\mathrm{HbA1c}$ increases in a dose dependent manner with increasing glucose concentration at room temperature. Despite supraphysiological glucose concentrations the reaction was slow. This is consistent with $\mathrm{HbA} 1 \mathrm{c}$ as a measure of average glucose concentration over 2-3 months ${ }^{2}$. The results from this in vitro incubation are also consistent with previous findings that persistent hyperglycemia over 2 weeks is needed in order to see increases in $\mathrm{HbA1c}$ in vivo ${ }^{13}$. 
351 Important components of a method validation study are imprecision and accuracy ${ }^{25}$. 352 Imprecision in the $\mathrm{DCA}^{\mathrm{TM}}$ Vantage was low with a CV of $2.97 \%$ for the normal commercial quality control. This is comparable to CVs obtained by several independent studies validating the DCA ${ }^{\mathrm{TM}}$ Vantage for use in humans where $<3.1 \%$ was achieved ${ }^{26} 27$. Slightly higher CVs were obtained during assessment of interassay variation using canine samples, mainly due to the low samples showing proportionally much more variation with an overall range of $1 \mathrm{mmol} / \mathrm{mol}$ compared to samples with a higher value. The overall imprecision is likely to be acceptable for veterinary use $\mathrm{e}^{28}$. A much higher inter-assay variation $(8.81 \%)$ was obtained from the high control. It is known that the $\mathrm{DCA}^{\mathrm{TM}}$ Vantage exhibits increased variance at readings above $64 \mathrm{mmol} / \mathrm{mol}^{26}$. However as only a small number of diabetic animals were included in the study (with a maximum $\mathrm{HbA} 1 \mathrm{c}$ of $48 \mathrm{mmol} / \mathrm{mol}$ ) it cannot be said whether this will impact veterinary use. Bias in the DCA $^{\mathrm{TM}}$ Vantage could not be assessed for canine $\mathrm{HbA} 1 \mathrm{c}$ measurements due to the absence of a canine reference method and the inaccessibility of previously validated methods. Comparison using high performance liquid chromatography set up for human $\mathrm{HbA} 1 \mathrm{c}$ has been unsuccessful in previous canine studies ${ }^{3,11,18}$. With the human reference material the abnormal control showed considerable bias compared to the reported mean of the material, but this was marginal in the normal control. The effect of storage at $4^{\circ} \mathrm{C}$ was minimal with a mean change from baseline of $-0.33 \mathrm{mmol} / \mathrm{mol}$ after 5 days. Different samples were used for the repeatability and storage assessments so variation cannot be compared directly, but the total variance from the storage assessment (CV 2.12\%) was comparable to the inter-assay variation.

The linearity study provided an indirect assessment of bias. The regression analysis confirmed that the line obtained experimentally was not significantly different from 
Validation of a method for measuring canine $\mathrm{HbA1c}$

$376 y=x$ and thus there was no internal bias against expected values. The results

377 obtained here are consistent with human data from independent studies ${ }^{19,26}$. The high $\mathrm{HbA1c}$ used in assessing linearity was significantly lower than the highest reading obtained from any animal in this study $(40 \mathrm{mmol} / \mathrm{mol} \mathrm{vs}$. $48 \mathrm{mmol} / \mathrm{mol})$, so there is potential for unknown bias in the upper expected working ranges of the assay and means that we have not fully established the reportable range of this assay. However as validation of this assay for human samples has established a reportable range of $<143 \mathrm{mmol} / \mathrm{mol}^{19}$ it is unlikely that any samples will exceed the reportable range when using the $\mathrm{DCA}^{\mathrm{TM}}$ Vantage for veterinary purposes. No significant difference was found between measurements obtained from blood anticoagulated with EDTA or heparin before analysis. The mean difference of $0.8 \mathrm{mmol} / \mathrm{mol}$ and lack of significant differences between the regression lines, suggests that these anticoagulants can be used interchangeably in the measurement of canine $\mathrm{HbA} 1 \mathrm{c}$.

In these preliminary investigations, hemolysis and hyperlipemia appear to interfere with the HbA1c measurement obtained by the $\mathrm{DCA}^{\mathrm{TM}}$ Vantage. The preliminary investigations were designed to test whether there was interference when using patient samples with the concentration range of interferent expected with clinical use, to determine where further investigation is warranted ${ }^{29}$. The manufacturers report that, for human samples, there is a bias of $-1.81 \%$ at triglyceride concentrations of $15.2 \mathrm{mmol} / \mathrm{L}$ for $\mathrm{HbA} 1 \mathrm{c}$ measurements in the $42-48 \mathrm{mmol} / \mathrm{mol}$ range, but no data on the magnitude of interference beyond this is available. As dogs may have triglyceride concentrations in excess of $15.2 \mathrm{mmol} / \mathrm{L}^{30}$, it was important to determine whether there may be an effect of hyperlipidemia on the HbA1c as measured by the device. 
401 from baseline in measured $\mathrm{HbA} 1 \mathrm{c}$ concentration after lipid removal that were larger 402 than the inherent imprecision within the method. It gives an early indication that

403

404

405

406

407

408

409

410

411

412

413

414

415

416

417

418

419

420

421

422

423

424 measured HbA1c may be artificially reduced in samples with gross hyperlipidemia and this should be investigated further using established guidelines ${ }^{21}$. Similarly, hemolyzing a pooled sample significantly increased the HbA1c reading obtained compared to baseline. This was surprising as the DCA ${ }^{\mathrm{TM}}$ Vantage hemolyzes the sample internally before analysis. So far only $100 \%$ hemolysis has been looked at and further investigation will be necessary to determine the maximum acceptable sample hemolysis. The other main interferent which is often looked at in validation studies is bilirubin, however unfortunately no sufficiently icteric samples were obtained during the course of the study.

The reference interval was $9-18.5 \mathrm{mmol} / \mathrm{mol}$ for canine $\mathrm{HbA} 1 \mathrm{c}$ in a hospital population. With an assay specific for canine HbA1c we would expect a lower reference interval compared to the human reference interval due to the decreased glucose permeability of canine erythrocytes compared to human erythrocytes ${ }^{1}$. The human reference interval is $20-42 \mathrm{mmol} / \mathrm{mol}$. As there is no canine reference method, our results cannot be readily compared to previous studies. When comparing our results to those obtained with previously evaluated methods for canine HbA1c still available for human use ${ }^{1617}$, our reference interval was significantly lower. As a point of note, one of the previous studies ${ }^{35}$ determined health based on the absence of clinical signs and did not specifically exclude anemic dogs. Anemic dogs were excluded in our study as there is evidence of an effect on canine $\mathrm{HbA} 1 \mathrm{c}$ independent of glucose concentration ${ }^{13} 1631$.

The hospital population may not mirror the true healthy population, despite evidence that most pathological processes do not affect $\mathrm{HbA} 1 \mathrm{c}^{13}$. A previous study did not 
show any differences between the reference interval obtained from a hospital sample

427

428

429

430

431

432

433

434

435

436

437

438

439

440

441

442

443

444

445

446

447

448

449

450 compared to healthy animals, but their healthy population consisted of 18 juvenile colony beagles ${ }^{3}$. The ethical issues involved with collecting samples from healthy animals for research purposes preclude the use of healthy reference animals ${ }^{32}$ (regulated under the Animals Scientific Procedures Act 1986). The reference interval is however relevant to dogs presented to a small animal hospital. Using this reference interval in other situations may not be appropriate. Sample size is another potential limitation in this study in that it reduced the power of the study to detect small differences between sub-groups. Despite a previous study with a larger sample size observing normality ${ }^{11}$, the distribution of reference values of $\mathrm{HbA1c}$ in our study was not normal (Figure 3). The reason for this distribution is not clear. The study was large enough, however, to establish a reference interval in line with American Society of Veterinary Clinical Pathology recommendations ${ }^{33}$ with good confidence in the range as shown by the tight $90 \%$ confidence intervals.

Information was specifically collected with a view to partitioning the reference population into subgroups. When performing subgroup analysis, the $\mathrm{HbA1c}$ was found to be significantly higher in the old non-predisposed group when compared to the rest of the reference interval, but a Kruskal-Wallis ANOVA identified no significant differences between the groups. There was no difference when partitioning solely by age (Figure 3), which is consistent with previous studies ${ }^{3,14}$. To our knowledge breed predisposition has not been previously examined in conjunction with $\mathrm{HbA1c}$. There was no significant effect of breed predisposition on HbA1c. However it is acknowledged that, as we only looked at predisposed compared to non-predisposed breeds, our categorization may have reduced the ability to detect a true difference in $\mathrm{HbA1c}$ in specific breeds or in the small sub- 
451 group of non-predisposed breeds that might be considered 'protected' against

452 diabetes mellitus. . Predisposed breeds are not likely to have a higher $\mathrm{HbA} 1 \mathrm{c}$ as the

453 clinical course of canine DM is comparable to human type 1 disease ${ }^{34}{ }^{35}$. However

454 as little is known about breed differences in erythrocyte permeability, but at least one

455 breed associated difference has been identified ${ }^{36}$, it follows that some breeds may

456 have higher (or lower) HbA1c concentrations.

457 In order for canine $\mathrm{HbA1c}$ to be adopted as an effective biomarker for long term

458 diabetic control, an independent objective assessment of diabetic control needs to

459 be developed. It will then be possible to assess the relative values of $\mathrm{HbA} 1 \mathrm{c}$ and 460 fructosamine as markers of long term diabetic control.

\section{Acknowledgements}

463 The authors would like to thank the staff at the Small Animal Hospital and the 464 Veterinary Diagnostics Service, University of Glasgow for their technical assistance. 465 We would also like to thank the University of Glasgow School of Veterinary Medicine 466 Fund for funding part of this study and Siemens Healthcare Plc for providing some of 467 the reagents. 
1. Higgins PJ, Garlick RL, Bunn HF. Glycosylated hemoglobin in human and animal red-cells - role of glucose permeability. Diabetes 1982;31(9):743-48.

2. Mortensen HB, Christophersen C. Glucosylation of human hemoglobin-a in redblood-cells studied in vitro - kinetics of the formation and dissociation of hemoglobin-a1c. Clin Chim Acta 1983;134(3):317-26.

3. Davison LJ, Podd SL, Ristic JME, Herrtage ME, Parnham A, Catchpole B. Evaluation of two point-of-care analysers for measurement of fructosamine or

4. Reusch CE, Haberer B. Evaluation of fructosamine in dogs and cats with hypo- or hyperproteinaemia, azotaemia, hyperlipidaemia and hyperbilirubinaemia. Vet Rec. 2001;148:370-3765. Little RR, Rohlfing CL. The long and winding road to optimal HbA1c measurement. Clin Chim Acta 2013;418:63-71.

6. The Diabetes Control and Complications Trial Research Group. The Effect of Intensive Treatment of Diabetes on the Development and Progression of Long-Term Complications in Insulin-Dependent Diabetes Mellitus. N Engl J Med 1993;329(14):977-86.

7. Stratton IM, Adler AI, Neil HAW, et al. Association of glycaemia with macrovascular and microvascular complications of type 2 diabetes (UKPDS 35): prospective observational study. BMJ 2000;321(7258):405-12.

8. Smith JE, Wood PA, Moore K. Evaluation of a colorimetric method for canine glycosylated hemoglobin. Am J Vet Res 1982;43(4):700-01.

9. Mahaffey EA, Cornelius LM. Evaluation of a commercial kit for measurement of glycosylated hemoglobin in canine blood. Vet Clin Pathol. 1981;10(1):21-24. 
10. Easley JR. Glycosylated hemoglobin in dogs: precision, stability, and diagnostic utility. Vet Clin Pathol. 1986;15(1):12-15.

11. Hooghuis $\mathrm{H}$, Rodriguez $\mathrm{M}$, Castano $\mathrm{M}$. lon-exchange microchromatography and thiobarbituric acid colorimetry for the measurement of canine glycated hemoglobins. Vet Clin Pathol.1994;23(4):110-16.

12. Jensen AL. Glycated blood proteins in canine diabetes-mellitus. Vet Rec 1995;137(16):401-05.

13. Haberer B, Reusch CE. Glycated haemoglobin in various pathological conditions: investigations based on a new, fully automated method. J Small Anim Pract 1998;39(11):510-17.

14. Marca MC, Loste A. Glycosylated haemoglobin assay of canine blood samples. J Small Anim Pract 2000;41(5):189-92.

15. Horauf A, Huder M, Reusch C. Evaluation of a fully automated method for the measurement of glycated hemoglobin in the dog. Tierarztl Prax Ausg $K$ Kleintiere Heimtiere 2000;28(5):345-48.

16. Catchpole B, Mountford S, Barabas S, Scaramuzzi RJ. Evaluation of a disposable device for the measurement of haemoglobin A1c in dogs. Vet Rec 2008;162(2):47-49.

17. Lien YH, Huang H-P. Glycosylated Hemoglobin Concentrations in Dogs with Hyperadrenocorticism and/or Diabetes Mellitus Compared to Clinically Healthy Dogs. Journal of Veterinary Clinical Sciences 2009;2(2):37-41.

18. Hasegawa S, Sako T, Takemura N, Koyama H, Motoyoshi S. Glycated hemoglobin fractions in normal and diabetic dogs measured by highperformance liquid-chromatography. J Vet Med Sci 1991;53(1):65-68. 
19. Szymezak J, Leroy N, Lavalard E, Gillery P. Evaluation of the DCA Vantage analyzer for HbA(1c) assay. Clin Chem Lab Med 2008;46(8):1195-98.

20. McEnroe RJ, Burrit MF, Powers DM, Rheinheimer DW, Wallace BH. CLSI: Interference Testing in Clinical Chemistry; Approved Guideline. Clinical and Laboratory Standards Institute document EP7-A2. Interference Testing in Clinical Chemistry. 2nd ed. Wayne, Pennsylvania, 2005.

21. Flatland B, Freeman KP, Friedrichs KR et al. ASVCP quality assurance guidelines: Control of general analytical factors in veterinary laboratories. Vet Clin Pathol 39(3) (2010) 264-277.

22. Geffre A, Concordet D, Braun J-P, Trumel C. Reference Value Advisor: a new freeware set of macroinstructions to calculate reference intervals with Microsoft Excel. Vet Clin Pathol .2011;40(1):107-12.

23. Spicer KM, Allen RC, Hallett D, Buse MG. Synthesis of hemoglobin-a1c and related minor hemoglobins by erythrocytes - invitro study of regulation. J Clin Invest 1979;64(1):40-48.

24. Cruz WO, Baumgarten A. Susceptibility of the red blood cell of the dog to haemolysis in alkaline media. Br J Haematol 1957;3(4):359-65.

25. Flatland B, Friedrichs KR, Klenner S. Differentiating between analytical and diagnostic performance evaluation with a focus on the method comparison study and identification of bias. Vet Clin Pathol .2014;43(4):475-86.

26. Lenters-Westra E, Slingerland RJ. Three of 7 Hemoglobin A(1c) Point-of-Care Instruments Do Not Meet Generally Accepted Analytical Performance Criteria. Clin Chem 2014;60(8):1062-72.

27. Lenters-Westra E, Slingerland RJ. Hemoglobin A1c Point-of-Care Assays; a New World with a Lot of Consequences! J Diabetes Sci Technol 2009;3(3):418-23. 
28. Harr KE, Flatland B, Nabity M, Freeman KP ASVCP guidelines: allowable total error guidelines for biochemistry. Vet Clin Pathol .2013;42(4):424-36.

29. Lumsden JH. Laboratory test method validation. Rev Med Vet (Toulouse) 2000;151(7):623-30.

30. Xenoulis PG, Levinski MD, Suchodolski JS, et al. Serum Triglyceride Concentrations in Miniature Schnauzers with and without a History of Probable Pancreatitis. J Vet Intern Med 2011;25(1):20-25.

31. Marca MC, Loste A, Unzueta A, Perez M. Blood glycated hemoglobin evaluation in sick dogs. Can J Vet Res 2000;64(2):141-44.

32. RCVS: Ethical Review for Practice-based Research: Advice on ethical review for veterinary surgeons planning clinical research. RVCS, 2013. Available at: http://www.rcvs.org.uk/publications/ethical-review-for-practice-basedresearch/. Accessed 23 August 2015.

33. Friedrichs KR, Harr KE, Freeman KP, et al. ASVCP reference interval guidelines: determination of de novo reference intervals in veterinary species and other related topics. Vet Clin Pathol .2012;41(4):441-53.

34. Davison LJ, Herrtage ME, Catchpole B. Study of 253 dogs in the United Kingdom with diabetes mellitus. Vet Rec 2005;156(15):467-+.471

35. Shields EJ, Lam CJ, Cox AR, et al. Extreme Beta-Cell Deficiency in Pancreata of Dogs with Canine Diabetes. PloS One 2015;10(6).

36. Conrado FO, Oliveira ST, Lacerda LA, Silva MOD, Hlavac N, Gonzalez FHD. Clinicopathologic and electrocardiographic features of Akita dogs with high and low erythrocyte potassium phenotypes. Vet Clin Pathol.2014;43(1):50-54.

37. Catchpole B, Ristic JM, Fleeman LM, Davison LJ. Canine diabetes mellitus: can old dogs teach us new tricks? Diabetologia 2005;48(10):1948-56. 
Validation of a method for measuring canine $\mathrm{HbA} 1 \mathrm{c}$

567

568

569

570

571

572

573

574

575

38. Mattin M, O'Neill D, Church D, McGreevy PD, Thomson PC, Brodbelt D. An epidemiological study of diabetes mellitus in dogs attending first opinion practice in the UK. Vet Rec 2014;174(14):349-+.352

39. Guptill L, Glickman L, Glickman N. Time trends and risk factors for diabetes mellitus in dogs: Analysis of Veterinary Medical Data Base records (19701999). Vet J 2003;165(3):240-47.

40. Fall T, Hamlin HH, Hedhammar A, Kampe O, Egenvall A. Diabetes mellitus in a population of 180,000 insured dogs: Incidence, survival, and breed distribution. J Vet Intern Med 2007;21(6):1209-16. 


\section{hyperlipidemia}

\begin{tabular}{|c|c|c|c|c|c|c|c|}
\hline$n^{1}$ & Diabetic & $\begin{array}{l}\text { total lipids }{ }^{2} \\
\text { (mmolL) }\end{array}$ & $\begin{array}{l}\text { TG }(\mathrm{RI}<0.6) \\
\text { (mmolL) }\end{array}$ & $\begin{array}{l}\text { cholesterol }(\mathrm{RI}<7) \\
(\mathrm{mmolL})\end{array}$ & $\begin{array}{l}\text { HbA1c pre } \\
\text { (mmol } / \mathrm{mol})\end{array}$ & $\begin{array}{l}\mathrm{HbA1c} \text { post }^{4} \\
(\mathrm{mmol} / \mathrm{mol})\end{array}$ & $\begin{array}{l}\text { Difference }^{5} \\
\text { (mmol/mol) }\end{array}$ \\
\hline 1 & & 5.62 & 0.68 & 4.94 & 15 & 15 & 0 \\
\hline 2 & Yes & 15.35 & 7.05 & 8.30 & 34 & 34 & 0 \\
\hline 3 & Yes & 19.55 & 9.26 & 10.29 & 13 & 13 & 0 \\
\hline 4 & & 21.59 & 11.64 & 9.95 & 13 & 14 & +1 \\
\hline 5 & Yes & 25.28 & 14.88 & 10.40 & 10 & 16 & +6 \\
\hline 6 & & 31.7 & 4.7 & 27.00 & 11 & 14 & +3 \\
\hline 7 & Yes & 36.65 & 25.58 & 11.07 & 29 & 35 & +6 \\
\hline
\end{tabular}

TG: Triglycerides, RI: Reference Interval. ${ }^{1}$ Sample number. ${ }^{2}$ Total measured lipids is defined as the sum of 


\begin{tabular}{|c|c|c|c|c|c|c|}
\hline \multirow[b]{4}{*}{ Category } & \multicolumn{4}{|c|}{ Subgroups of the reference population } & \multirow{3}{*}{$\begin{array}{r}\text { Total } \\
\text { Reference } \\
\text { population }\end{array}$} & \multirow{3}{*}{$\begin{array}{r}\text { diabetic } \\
\text { group }\end{array}$} \\
\hline & \multirow{2}{*}{$\begin{array}{l}\text { young non- } \\
\text { pre disposed }\end{array}$} & \multirow{2}{*}{$\begin{array}{l}\text { old non-pre } \\
\text { disposed }\end{array}$} & \multirow{2}{*}{$\begin{array}{l}\text { young pre } \\
\text { disposed }\end{array}$} & \multirow{2}{*}{$\begin{array}{l}\text { old pre } \\
\text { disposed }\end{array}$} & & \\
\hline & & & & & & \\
\hline & $n=20$ & $\mathrm{n}=18$ & $n=9$ & $n=13$ & $n=60$ & $n=10$ \\
\hline Age Mean (SD) & $3.25(1.29)$ & $9.28(2.45)$ & $2.11(1.27)$ & $8.85(1.63)$ & $6.1(3.60)$ & $10.10(3.96)$ \\
\hline Median & 4 & 9 & 2 & 9 & 5 & 10.5 \\
\hline Range & $1-5$ & $6-14$ & $1-4$ & $6-11$ & $1-14$ & $1-14$ \\
\hline \multicolumn{7}{|l|}{ Sex } \\
\hline male entire $n(\%)$ & $5(25 \%)$ & $8(44.4 \%)$ & $4(44.4 \%)$ & $1(7.7 \%)$ & $18(30 \%)$ & $1(10 \%)$ \\
\hline male neut $n(\%)$ & $1(5 \%)$ & $4(22.2 \%)$ & $1(11.1 \%)$ & $4(30.8 \%)$ & $10(16.7 \%)$ & $5(50 \%)$ \\
\hline female entire $n(\%)$ & $5(25 \%)$ & $2(11.1 \%)$ & $0(0 \%)$ & $3(23.1 \%)$ & $10(16.7 \%)$ & $1(10 \%)$ \\
\hline female neut $\mathrm{n}(\%)$ & $9(45 \%)$ & $4(22.2 \%)$ & $4(44.4 \%)$ & $5(38.5 \%)$ & $22(36.7 \%)$ & $3(30 \%)$ \\
\hline \multicolumn{7}{|l|}{ Breed predisposition } \\
\hline Yes n(\%) & & & & & $22(36.7 \%)$ & $7(70 \%)$ \\
\hline no $n(\%)$ & & & & & $38(63.3 \%)$ & $3(30 \%)$ \\
\hline \multicolumn{7}{|l|}{ Blood glucose (mmolL) } \\
\hline Mean (SD) & $4.83(0.469)$ & $4.96(0.544)$ & $5.18(0.954)$ & 4.94 (0.549) & $4.9(0.596)$ & $19.56(10.6)$ \\
\hline Median & 4.9 & 5 & 5.3 & 4.9 & 4.9 & 19.3 \\
\hline Range & $3.8-5.9$ & $3.7-5.8$ & $3.6-6.8$ & $4.1-5.9$ & $3.6-6.8$ & $3.9-35.1$ \\
\hline \multicolumn{7}{|l|}{ Hemoglobin (g/dL) } \\
\hline Mean (SD) & $15.98(2.32)$ & $15.51(1.80)$ & $15.77(1.71)$ & $15.78(3.25)$ & $15.8(2.29)$ & $15.09(1.43)$ \\
\hline Median & 15.8 & 15.15 & 16.1 & 15.5 & 15.7 & 15.1 \\
\hline Range & $13-20.3$ & $12.6-19.2$ & $12.6-17.9$ & $11-23.2$ & $11-23.2$ & $13.4-17.5$ \\
\hline \multicolumn{7}{|l|}{$\mathrm{HbA} 1 \mathrm{c}(\mathrm{mmol} / \mathrm{mol})$} \\
\hline Mean (SD) & $14.1(2.5)$ & $15.28(1.97)$ & $13.89(2.32)$ & $13.62(3.20)$ & $14.3(2.5)$ & $36.5(9.94)$ \\
\hline Median & 13.5 & 15.5 & 13 & 13 & 14 & 39 \\
\hline
\end{tabular}


601

602

603

604

605

606

607

608

Figure Legends

Figure 1: Change in HbA1c in hemolysates incubated with increasing concentrations of glucose at room temperature. $\$ 400 \mathrm{mM}$ glucose, $\nabla 200 \mathrm{mM}$ glucose, $\triangle 100 \mathrm{mM}$ glucose, $=50 \mathrm{mM}$ glucose, $\bullet 0 \mathrm{mM}$ glucose.

Figure 2: Scatter plot of expected HbA1c values against observed HbA1c measurements in $\mathrm{mmol} / \mathrm{mol}$ using 2 baseline samples and 3 intermediate mixes(25:75, 50:50, 75:25) using EDTA (squares) and heparin-anticoagulated blood (triangles) blood. Straight lines are regression lines; dotted line is $y=x$.

Figure 3: Histogram of all reference values $(n=60)$ partitioned by age (left panel) and breed disposition (right panel).

Figure 4: Boxplot of $\mathrm{HbA1C}$ values of the reference group compared to the diabetic group. The box represents the interquartile range and is bisected by a line representing the median. The lines represent the main body of data with open circles representing outlying points. 